\title{
Análise estatística implicativa da tendência de abordagens do método de estudo de casos no ensino de ciências ${ }^{1}$
}

Implicative statistical analysis of the trend to applying the case study method in science teaching

\author{
Ladjane Pereira da Silva Rufino de Freitas ${ }^{2}$ \\ Angela Fernandes Campos ${ }^{3}$ \\ Vladimir Lira Veras Xavier de Andrade ${ }^{4}$ \\ Jean-Claude Régnier ${ }^{5}$
}

\section{Resumo}

Este estudo realizou uma análise da tendência de abordagens do método de estudo de casos no ensino das ciências, com especificação para o ensino de Química. Para tanto, realizou-se uma análise estatística implicativa em 71 volumes de oito periódicos de divulgação científica no campo da Educação em Ciências, no período de 2005-2015. A análise nos possibilitou observar importantes relações hierárquicas implicativas nas tendências de abordagem do estudo de casos nos trabalhos investigados, onde as áreas de conhecimento da Biologia e interdisciplinaridade aparecem mais nos trabalhos que utilizam o estudo de casos aplicado ao ensino, estando esse tipo de aplicação mais presente nos periódicos estrangeiros e no ensino superior, já a Matemática, Física e Ciências aparecem mais nos trabalhos que utilizam o método aplicado à pesquisa, com a Química na interface dessas duas utilizações. Estando ainda essa última utilização do método mais presente nos periódicos nacionais e no ensino básico. No ensino de Química o estudo realizado sugere a necessidade de implementação de práticas pedagógicas que utilizem a metodologia de estudo de casos em diferentes níveis de ensino.

Palavras-chave: estudo de caso; análise estatística; ensino das ciências.

\section{Abstract}

This study conducted an analysis of the trend to applying the case study method in science teaching, specifically chemistry. To that end, an implicative statistical analysis was carried out in 71 issues of Science Education Journals between 2005 and 2015. Analysis revealed important implicative hierarchical relations in the trends to case studies in the articles investigated, where the biological and interdisciplinary sciences appear more in papers that use case studies applied to teaching. This type of application is more present in foreign

\footnotetext{
1 Pesquisa realizada com o apoio financeiro da CAPES através da bolsa PVE.

2 Universidade Federal de Campina Grande | ladjanepsbr@yahoo.com.br

3 Universidade Federal Rural de Pernambuco | afernandescampos@gmail.com

4 Universidade Federal Rural de Pernambuco | vladiandrade@gmail.com

5 Université de Lyon, France | jean-claude.regnier@univ-lyon2.fr
} 
journals and in higher learning, with mathematics, physics, and sciences more prevalent in studies that apply the method to research, and chemistry on the interface of these two applications. The latter use of the method is more common in national journals and elementary education. For chemistry teaching, the present study suggests the need to implement pedagogic practices that use the case study methodology at different teaching levels.

Keywords: Case Studies, Implicative Statistical analysis, Science Teaching.

\section{Introdução}

O método de estudo de casos, segundo Martins (2008) trata-se de uma das mais antigas formas de investigação científica conhecida e cuja aplicação extrapola sua seara original, chegando a campos como o jornalismo, a administração, a contabilidade, a economia e a educação.

Em termos de abordagem, segundo Bocchi et al. (1996), o estudo de casos pode ser discutido sob dois enfoques: como modalidade de pesquisa e como estratégia de ensino.

Como modalidade de pesquisa, o método de estudo de casos se constitui numa investigação que envolve a análise de um fenômeno em profundidade dentro do seu contexto, podendo ainda ser utilizado para testar hipóteses, como também pode se constituir num relato de pesquisa investigativa e diagnóstica, entre outras aplicações (YIN, 2001, p. 21).

Como estratégia de ensino, segundo Sá e Queiroz (2010, p.12) "é um método que oferece aos estudantes a oportunidade de direcionar sua própria aprendizagem e investigar aspectos científicos e sócio-científicos, presentes em situações reais ou simuladas e de complexidade variável".

O estudo de casos aplicado ao ensino teve sua origem na faculdade de direito de Harvard University por volta de 1870. No final do século XIX, Christopher Collumbus Langdell fundador do método, revolucionou a escola de direito de Harvard, uma vez que esse método tornou possível o estudo prático da lei (CONANT, 1968; ELLET, 2008). De acordo com Menezes (2009, p.137) "era o estudo da lei viva. A partir da análise dos arrazoados dos juízes os alunos iam chegando às próprias conclusões sobre a legislação". 0 sucesso do método nos cursos de direitos fez com que, paulatinamente, o método fosse estendido a outros campos do conhecimento como Administração, Medicina e Educação (BOAVENTURA, 2014).

Tempos depois, na década de 1910, o método chega a Harvard Business School, a reputada faculdade de administração de empresas, considerada hoje a maior produtora de "casos" e materiais de treinamento para o uso do método em sala de aula (SILVA; BENEGAS, 2010). Na Administração, os casos, também conhecidos como 'Casos comerciais', apresentam as experiências das empresas em suas trajetórias e aplicabilidade. Os casos são utilizados pelos professores para levar os estudantes a refletirem e analisarem situações reais (MENEZES, 2009).

Na Medicina, os 'Casos Clínicos' como são assim conhecidos, tem por finalidade proporcionar aos seus estudantes o contato com problemas reais para a aprendizagem dos conteúdos estudados (HERREID, 1997). Casos de pacientes são tomados como situações reais analisadas pelos estudantes. Essa forma de abordagem também recebe o nome de análise de casos. 
Por muito tempo o método de estudo de casos como estratégia de ensino ficou restrito à formação de profissionais da área de medicina, direito e administração, porém, nessas últimas décadas sua introdução também ocorre na área de Educação em Ciências (HERREID, 2005).

Quanto a sua utilização no Ensino das Ciências, em 1994, Herreid publicou um artigo intitulado "Case studies in science - a novel method of science education" no Journal of College Science Teaching, que deu origem à seção denominada "The Case Study". Nessa seção são publicados artigos com diferentes propostas de aplicação e avaliação do método com o intuito de auxiliar o professor quanto à utilização dos casos em sala de aula.

De acordo com Herreid (1994), James B. Conant, professor de Harvard, foi o primeiro educador em ciência a organizar um curso inteiro em torno desta modalidade de ensino. Daí em diante, os professores de Harvard começaram a introduzir casos pela primeira vez para dar aos estudantes experiência prática para uso no mundo real.

Com o intuito de aumentar a divulgação da utilização do método de estudo de casos no ensino de Química, em 1998, o periódico Journal of Chemical Education criou uma seção específica para o assunto, intitulada "Teaching With Problems and Case Studies". A criação da mesma foi justificada pelos editores da revista, por naquele momento, existirem poucos relatos de casos elaborados especificamente para utilização no ensino de Química (SÁ, 2006).

No Brasil, o grupo de Pesquisa em Ensino de Química do Instituto de Química de São Carlos (GPEQSC) da Universidade de São Paulo foi o pioneiro a introduzir o método de estudo de casos no ensino das Ciências, o grupo produz casos abordando questões em temas sócio-científicos e científicos.

Os casos em geral, destinados ao ensino das Ciências, apresentam em seus contextos questões sociais, ambientais, econômicas e éticas, para estimular no estudante a capacidade de tomar decisões diante de problemas da vida real e de argumentar a favor das mesmas, além de promoverem uma melhor compreensão da natureza da ciência, de suas potencialidades e limitações, preparando os alunos para uma intervenção mais crítica em discussões e debates públicos sobre questões sócio-científicas. (REIS, 2007; SÁ; QUEIROZ, 2010).

\section{O estudo de casos e suas abordagens: pesquisa versus ensino}

Alguns trabalhos como os de Ikeda (2005); Menezes (2009); Moreira (2011); ClementeJúnior (2012); Vasconcelos et. al. (2013) e Carniello (2015), entre outros, procuram elucidar as diferenças entre esses dois tipos de abordagens do método de estudo de casos, pois, de acordo com esses autores, normalmente tanto estudantes como pesquisadores iniciantes, confundem as suas abordagens. Vasconcelos et. al. (2013), são uns dos que explicitam essa problemática ao colocar que, apesar das diferenças, ainda é muito comum a abordagem do método de estudo de casos ser confundida na prática docente, embora possuam conceitos distintos.

Para facilitar a diferenciação entre essas duas abordagens do método, alguns autores passaram a denominar o método de estudo de casos com abordagem para o ensinoaprendizado como "método do caso". Apesar do novo termo, muitos desses autores, para evitar que ainda persista algum tipo de dúvida, ao discorrer sobre o método e sua abordagem para o ensino, procuram sempre deixar claro essa diferença. 
Para Ikeda (2005), enquanto o estudo de caso é uma técnica de pesquisa qualitativa, que tem as atenções voltadas para o diagnóstico de um "caso", o método do caso, apresenta finalidades pedagógicas e serve, sobretudo, para ilustrar conceitos e desenvolver habilidades nos estudantes.

De acordo com Yin (1989), o estudo de casos para fins de ensino não precisa, necessariamente, conter uma interpretação de eventos reais, pois, seu objetivo é de estabelecer uma estrutura de discussão e debate entre os alunos. Para o autor, os critérios que definem o desenvolvimento de casos para o ensino são muito diferentes daqueles para pesquisa, os estudos de caso para o ensino não precisam estar preocupados com a apresentação rigorosa de dados empíricos, já os estudos de casos de pesquisa precisam.

Existem outras duas particularidades, além do termo, que contribuem para que seja gerada essa confusão: uma, é o fato do método de estudo de casos como modalidade de pesquisa, recentemente vir sendo utilizado na pesquisa em educação com o objetivo de conduzir um estudo descritivo de uma unidade, que tanto pode ser uma escola, um professor, uma aula, como um currículo ou um sistema escolar (MOREIRA, 2011; ANDRÉ, 1998). A outra particularidade é a utilização dos casos de pesquisa no ensino, nesse contexto, os casos que antes se caracterizavam como investigativos, quando levados para a sala de aula para serem usados como dilema para a problematização de conceitos ou de temas, passam a se caracterizar como casos de ensino. Neste sentido, Vasconcelos et. al. (2013, p. 8) ao estabelecerem diferenças entres estas duas abordagens, colocam que "não obstante se advogue que ambas podem ser empregadas como estratégia didáticopedagógica".

A dualidade de abordagens do método de estudo de casos, nos levou a investigar o seguinte problema de pesquisa: Que tendências de abordagens têm caracterizado a utilização do método do estudo de casos em trabalhos científicos publicados na área de Educação em Ciências, especialmente no ensino de Química?

Com o intuito de responder a esse problema, esta pesquisa teve por objetivo elucidar, por meio da literatura, a tendência de abordagem do método de estudo de casos predominante em trabalhos publicados no período de 2005 a 2015 na literatura nacional e estrangeira voltada para o ensino das Ciências.

\section{Metodologia da pesquisa}

Esta investigação se constituiu numa pesquisa teórica, bibliográfica de natureza qualitativa exploratório-descritiva. $O$ enfoque desta pesquisa apesar de ter priorizado aspectos qualitativos, também teve sua interface na quantitativa, pois trabalhos como os de Moreira (2011); Boaventura (2014) e André, (2007) mostram que os métodos qualitativos e quantitativos na maior parte das pesquisas em educação tem se complementado e que a pesquisa nessa área pode ser feita sob qualquer um desses dois enfoques. Para esses autores, a pesquisa em educação pode se utilizar de uma abordagem quantitativa ou qualitativa ou ainda de ambas, desde que se chegue à resolução do problema. Também, é válido ressaltar que pesquisas de temáticas específicas relacionadas ao ensino de Ciências são importantes, pois, além de fornecerem tendências atuais concernentes as temáticas escolhidas, possibilitam um olhar sobre possíveis desdobramentos de novas pesquisas e linhas de investigação (BARBOSA, 2018). 


\section{Seleção da amostra e justificativa da escolha}

Para identificarmos tendências de abordagem do método de estudo de casos, buscamos fazer um levantamento bibliográfico de trabalhos publicados no período de 2005 a 2015 em 03 (três) dos principais periódicos estrangeiros de publicação científica da área de Educação em Ciências e 05 (cinco) periódicos nacionais.

De acordo com critérios por nós pré-definidos, os três periódicos estrangeiros escolhidos foram: o Journal Chemical Education, o Chemistry Education Research Practice e o Journal College Science Teaching.

O Journal Chemical Education e a Chemistry Education Research Practice foram escolhidos por serem específicos da área de Química, por se tratarem de periódicos científicos com grande circulação internacional e por gozarem de um bom conceito na área, com estratificação A pela CAPES e fator de impacto. Já o Journal College Science Teaching foi escolhido por possuir uma coluna exclusiva voltada para artigos que discutem inovações no ensino pelo estudo de casos e avaliações do método, além disso, por ser um periódico de publicação da National Science Teachers Association, considerada uma das maiores organizações do mundo, empenhada em promover a excelência e a inovação no ensino na área da Educação em Ciências.

Concernente às publicações nacionais, os cinco periódicos escolhidos foram: a revista Investigações em Ensino de Ciências, revista Ciência \& Educação, Revista Brasileira de Pesquisa em Educação em Ciências, revista Química Nova na Escola e a revista Experiências em Ensino de Ciências.

Os critérios pré-estabelecidos para a escolha destes periódicos foram: que tivessem suas publicações voltadas para o campo da Educação em Ciências; que possuíssem estratificação pela CAPES; frequência de publicação de no mínimo quadrimestral e que para o período pesquisado apresentassem no mínimo 15 trabalhos com publicação voltada para o tema "estudo de casos".

Os periódicos, Investigações em Ensino de Ciências, Ciência \& Educação e a Revista Brasileira de Pesquisa em Educação em Ciências, foram escolhidos por possuírem um bom conceito na área de Educação em Ciências e estratificação A pela CAPES, já os periódicos Experiências em Ensino de Ciências e Química Nova na Escola, apesar de apresentarem estratificação B1 pela CAPES, são periódicos que mais publicam relatos de estudos voltados para a Educação Básica e, além disso, a Química Nova na Escola é considerada a revista científica de maior circulação nas escolas de ensino básico.

As bases de dados para consulta destes periódicos foram: Portal de Periódicos da CAPES, a Webofscience e o Portal da SciElo.

\section{Coleta de dados}

A busca pelos trabalhos foi realizada utilizando descritores, palavras-chave e assuntos ('estudo de casos', 'casos', 'case study', 'case', 'estudio de caso') em consonância com o tema pesquisado. Foram feitas leituras dos resumos dos trabalhos encontrados e quando necessário a leitura do trabalho na íntegra. Foram considerados todos os trabalhos na área de Educação em Ciências que indicassem alguma relação de uso do tema pesquisado. 


\section{Tratamento dos dados}

A análise estatística implicativa (ASI) foi utilizada como ferramenta no tratamento dos dados deste trabalho por nos permitir visualizar, organizar, construir modelos e explicar fenômenos associados aos dados (GRAS; ALMOULOUD, 2003), além de que, de acordo com Almouloud, Gras e Régnier (2014, p.625) "a Análise Estatística Implicativa, tem por objetivo a extração de conhecimentos, invariantes, e atribuição de uma medida para proposições do tipo: 'quando A está escolhido, tende-se a escolher $\mathrm{B}^{\prime}$, .".

Com base nessas argumentações, acreditamos que a aplicação dessa análise nos permitiria observar importantes relações hierárquicas implicativas nas formas de abordagens do método do estudo de casos pelos trabalhos aqui investigados, pois, de acordo com Kerlinger (1980 apud MOREIRA, 2011, p. 30) "a estatística é a teoria e o método de analisar dados obtidos de amostras de observações com o fim de descrever populações, estudar e comparar fontes de variância para ajudar a tomar decisões sobre aceitar ou rejeitar relações entre fenômenos e para ajudar a fazer inferências fidedignas de operações empíricas".

A utilização da ASI, neste trabalho, foi feita com auxílio do software CHIC ${ }^{\circledR}$ (Versão 6.0, 2012) que é um software de análise de dados, sua sigla significa Classificação Hierárquica Implicativa e Coesiva, foi originalmente concebido por Régis Gras sobre os algoritmos e, em seguida, desenvolvido para PC por Saddo Ag Almouloud, Harrisson Ratsimba-Rajohn e na sua versão atual por Raphaël Couturier.

Segundo Couturier, Bodin e Gras (2004, p 1), o software CHIC tem por principais funções "extrair de um conjunto de dados, cruzando sujeitos e variáveis (ou atributos), regras de associação entre variáveis, fornecer um índice de qualidade de associação e de representar uma estruturação das variáveis obtida por meio destas regras".

Neste trabalho, os dados foram transformados em termos indicadores e no CHIC foram inseridos como variáveis binárias, que compreendeu unicamente dois valores, 0 ou 1 representando, respectivamente, a ausência e a presença da variável para cada indivíduo pesquisado.

As variáveis foram qualificadas como principais e suplementares: Como padrão, as variáveis normalmente consideradas pelo CHIC são as principais. As variáveis suplementares são consideradas apenas para efeito da contribuição.

Para efeito de tratamento dos dados pelo $\mathrm{CHIC}$, foram inseridos como objetos de análise os oitos periódicos com seus volumes analisados, totalizando 71 objetos. A tabela 1 apresenta os volumes analisados de cada periódico e suas abreviações usadas no CHIC.

Tabela 1. Volumes analisados de cada periódico e suas abreviações usadas no CHIC.

\begin{tabular}{l|l|l|c}
\hline \multicolumn{1}{c|}{ PERIÓDICOS } & \multicolumn{1}{c}{ ABREVIAÇÃO } & \multicolumn{1}{c}{ VOLUMES (V) } & TOTAL \\
\hline Journal Chemical Education & JChemEdu & $82 ; 84-92$ & 10 \\
Journal College Science Teaching & JCollSciTeach & $35-44$ & 11 \\
Chemistry Education Research Practice & ChemResPract & $8-16$ & 9 \\
Ciência \& Educação & CiêncEduc & $13-16 ; 18-21$ & 8 \\
Química Nova na Escola & QNEsC & $30 ; 31 ; 33-37$ & 7 \\
Investigações em Ensino de Ciências & IENCl & $10-15 ; 17-19$ & 9 \\
Experiências em Ensino de Ciências & EENCl & $1-3 ; 5-10$ & 9 \\
Revista Bras. de Pesquisa em Educ. em Ciências & RBPEC & $5-7 ; 9 ; 10 ; 12 ; 13 ; 15$ & 8 \\
\hline TOTAL GERAL DE VOLUMES & & 71 \\
\hline
\end{tabular}

Quadro 1. Apresentação das variáveis principais. 
Para efeito de interpretação colocamos as variáveis principais de acordo com as categorias pesquisadas, presentes no quadro 1.

\begin{tabular}{c|l}
\hline CATEGORIAS PESQUISADAS & \multicolumn{1}{c}{ VARIÁVEIS PRINCIPAIS } \\
\hline Origem do periódico & Estrangeira; Nacional \\
\hline Tipo de abordagem do método & Estratégia de Ensino; Modalidade de pesquisa; Apenas indicam \\
\hline Autores mais citados nos trabalhos & Autores Grupo 1; Autores Grupo 2 \\
\hline Nível de ensino & Ensino Superior; Ensino Básico \\
\hline Área de conhecimento & Química; Física; Matemática; Biologia; Ciências; Interdisciplinar \\
\hline
\end{tabular}

A seguir, apresentamos uma descrição mais detalhada de cada uma destas categorias:

Origem de periódico - Classificamos como 'nacional' os periódicos com edição brasileira e 'estrangeira' os periódicos com edição não brasileira.

Tipo de abordagem do método - Nesta categoria classificamos em 'Estratégia de Ensino' quando os autores utilizam claramente o método como uma estratégia de ensinoaprendizagem; 'Modalidade de pesquisa' quando os autores informam em seus trabalhos que o estudo de caso empregado se trata de uma forma de pesquisa e 'Apenas indicam' quando os autores apenas indicam que se trata de um estudo de caso, mas não fazem nenhuma referência ao método, em alguns casos o termo aparece apenas no título do trabalho ou apenas citado no resumo ou na metodologia. Essa classificação de abordagem do método do estudo de caso foi realizada em todos os trabalhos encontrados.

Autores mais citados nos trabalhos - Os 'autores grupo 1' foram os autores (Stake; Yin; Bogdan e Biklen; Godoy; Trivinos; Andre e Ludke) que mais foram citados nos trabalhos que apresentaram a abordagem do método do estudo de caso voltada para pesquisa, já os 'autores do grupo 2' foram os autores (Herreid; Conant; Sá e Queiroz; Kortland; Cornely; Cheng; Fortier) que mais foram citados nos trabalhos que apresentaram a abordagem do método do estudo de caso voltada para estratégia de ensino. Nesses dois grupos estão presentes os autores que formam o referencial teórico que fundamenta cada uma dessas duas abordagens do método.

Nível de ensino - Classificamos em 'Ensino Superior' todos os trabalhos que tiveram a abordagem do método voltada para a Graduação e Pós-Graduação, e 'Ensino Básico' todos os trabalhos que tiveram a abordagem do método voltada para o Ensino Médio e Fundamental.

Área de conhecimento - Área de saberes no qual os autores dos trabalhos investigados focaram seus estudos.

Com o auxílio do software CHIC realizamos os seguintes tratamentos de dados:

\section{Analise de Similaridade}

Efetuamos a análise de similaridade segundo proposto por Lerman (1980), que desenvolvida através da aplicação do software CHIC nos possibilitou a visualização das semelhanças e as proximidades entre os grupos apresentados de forma hierárquica através da árvore de similaridade.

Podemos definir a similaridade a partir do cruzamento do conjunto das variáveis com um conjunto de sujeitos (volumes). Este tipo de análise nos permite estudar e interpretar, em termos de tipologia e de semelhança (e não semelhança) decrescente, classes de variáveis, constituídas significativamente a certos níveis da árvore e se opondo a outras nestes mesmos níveis (GRAS; ALMOULOUD, 2003). 


\section{Gráfico implicativo}

O gráfico implicativo efetua os cálculos dos índices de implicação no sentido da análise implicativa, clássica ou entrópica, segundo a opção escolhida (COUTURIER, BODIN E GRAS, 2004).

\section{Resultados e discussão}

Quanto aos resultados da pesquisa, a seguir são apresentados os trabalhos encontrados nos veículos por nós analisados. Conforme mostra a tabela 2, foram encontrados 283 trabalhos que possuíam algum indicativo de abordagem do método de estudo de casos.

Tabela 2. Trabalhos encontrados relacionados ao uso do método de Estudo de Casos em Ciências.

\begin{tabular}{|c|c|c|c|c|c|c|c|c|}
\hline \multirow[b]{2}{*}{ ANO } & \multicolumn{3}{|c|}{ PERIÓDICOS ESTRANGEIROS } & \multicolumn{5}{|c|}{ PERIÓDICOS NACIONAIS } \\
\hline & $\begin{array}{l}\text { J. Chem. } \\
\text { Educ. }\end{array}$ & $\begin{array}{l}\text { J. Coll. Sci. } \\
\text { Teach }\end{array}$ & $\begin{array}{l}\text { Chem. Educ. } \\
\text { Res. Prat. }\end{array}$ & $\begin{array}{l}\text { Ciênc. } \\
\text { Educ. }\end{array}$ & QNEsC & INECI & EENCl & RBPEC \\
\hline 2005 & 02 & 12 & 00 & 00 & 00 & 02 & 00 & 01 \\
\hline 2006 & 00 & 14 & 00 & 00 & 00 & 01 & 02 & 01 \\
\hline 2007 & 02 & 11 & 02 & 01 & 00 & 03 & 01 & 02 \\
\hline 2008 & 04 & 02 & 06 & 03 & 02 & 03 & 01 & 00 \\
\hline 2009 & 03 & 04 & 03 & 02 & 02 & 02 & 00 & 01 \\
\hline 2010 & 05 & 06 & 02 & 04 & 00 & 02 & 03 & 03 \\
\hline 2011 & 05 & 03 & 03 & 00 & 02 & 00 & 03 & 00 \\
\hline 2012 & 06 & 05 & 03 & 02 & 03 & 07 & 05 & 02 \\
\hline 2013 & 14 & 08 & 06 & 04 & 02 & 03 & 04 & 01 \\
\hline 2014 & 07 & 09 & 01 & 02 & 01 & 02 & 04 & 00 \\
\hline 2015 & 14 & 07 & 07 & 06 & 05 & 00 & 01 & 05 \\
\hline \multirow{2}{*}{ Total } & 62 & 81 & 33 & 24 & 17 & 25 & 24 & 16 \\
\hline & \multicolumn{3}{|c|}{176} & \multicolumn{5}{|c|}{106} \\
\hline Total Geral & \multicolumn{8}{|c|}{283} \\
\hline
\end{tabular}

Ao analisarmos a quantidade de trabalhos publicados no Journal Chemical Education e no Journal College Scince Teaching, percebemos um número bastante expressivo de trabalhos se comparados com a quantidade de trabalhos encontrados nos periódicos nacionais.

Queremos salientar que nesses 283 trabalhos estão presentes os dois tipos de abordagem do método de Estudo de Casos. A abordagem do método como modalidade de pesquisa e como estratégia de ensino, conforme vimos nas colocações feitas por Bocchi et al. (1996) ao afirmarem a que o método de estudo de casos pode ser discutido sob esses enfoques.

Desses 283 trabalhos encontrados, 147 abordam o método de estudo de casos como estratégia de ensino, porém, desses 147 trabalhos, 128 são dos periódicos estrangeiros, conforme vemos na tabela 3 e apenas 19 são dos periódicos nacionais, ora investigados. 
Tabela 3. Trabalhos que abordam o método de Estudo de Casos como estratégia de ensino

\begin{tabular}{|c|c|c|c|c|c|c|c|c|c|}
\hline \multirow[b]{2}{*}{ Ano } & \multicolumn{3}{|c|}{ PERIÓDICOS ESTRANGEIROS } & \multicolumn{5}{|c|}{ PERIÓDICOS NACIONAIS } & \multirow{2}{*}{$\begin{array}{l}\text { Total } \\
\text { Ano }\end{array}$} \\
\hline & $\begin{array}{l}\text { J. Chem. } \\
\text { Educ. }\end{array}$ & $\begin{array}{l}\text { J. Coll. Sci. } \\
\text { Teach }\end{array}$ & $\begin{array}{c}\text { Chem. Educ. } \\
\text { Res. Prat. }\end{array}$ & $\begin{array}{l}\text { Ciênc. } \\
\text { Educ. }\end{array}$ & QNESC & INECI & EENCI & RBPEC & \\
\hline 2005 & 02 & 12 & 00 & 00 & 00 & 00 & 00 & 00 & 14 \\
\hline 2006 & 00 & 13 & 00 & 00 & 00 & 00 & 00 & 00 & 13 \\
\hline 2007 & 02 & 11 & 00 & 01 & 00 & 00 & 00 & 00 & 14 \\
\hline 2008 & 03 & 02 & 00 & 01 & 00 & 00 & 00 & 00 & 06 \\
\hline 2009 & 03 & 04 & 00 & 00 & 00 & 00 & 00 & 01 & 08 \\
\hline 2010 & 04 & 06 & 02 & 00 & 00 & 00 & 00 & 00 & 12 \\
\hline 2011 & 02 & 03 & 00 & 00 & 01 & 00 & 00 & 00 & 06 \\
\hline 2012 & 04 & 05 & 00 & 00 & 01 & 02 & 01 & 00 & 13 \\
\hline 2013 & 10 & 08 & 02 & 00 & 00 & 00 & 03 & 00 & 23 \\
\hline 2014 & 03 & 09 & 00 & 01 & 00 & 00 & 00 & 00 & 13 \\
\hline 2015 & 08 & 07 & 03 & 01 & 05 & 00 & 01 & 00 & 25 \\
\hline Total & 41 & 80 & 07 & 04 & 07 & 02 & 05 & 01 & \multirow{2}{*}{147} \\
\hline Total Geral & \multicolumn{3}{|c|}{128} & & & 19 & & & \\
\hline
\end{tabular}

\section{Análise dos trabalhos encontrados nos periódicos estrangeiros}

De acordo com a nossa pesquisa tanto o Journal College Science Teaching como o Journal Chemical Education apresentaram um alto índice de trabalhos publicados com utilização do método voltado para estratégia de ensino, com 99\% e 66\% dos trabalhos, respectivamente. Já a revista Chemistry Education Research Practice apresentou apenas 21\% do total de seus trabalhos com abordagem do método voltada para ensino, apresentou ainda um expressivo número de trabalhos com abordagem voltada para Modalidade de Pesquisa com 70\% de seus trabalhos e 9\% que apenas indicam o uso do método.

Quanto a área de conhecimento, dos 80 trabalhos publicados no Journal College Science Teaching que apresentaram o estudo de casos voltado para o ensino, 53\% são da área da interdisciplinaridade e $38 \%$ da Biologia. Talvez essa ocorrência se dê por essas duas áreas de estudo comporem a grade curricular dos cursos de medicina, onde o método de estudos de casos com essa abordagem possui uma antiga e vasta aplicação (HERREID, 1994).

As demais áreas de conhecimento como Química, Física e Ciências, compõem o restante, 9\%, dos trabalhos que abordam o método com estratégia de ensino.

Ao analisarmos esses resultados de acordo com os achados da literatura, podemos justificar que essa quantidade de trabalhos se deve aos seguintes fatores: primeiro, tanto o Journal Chemical Education, quanto o Journal College Science Teaching foram os pioneiros na publicação de trabalhos envolvendo a abordagem do método de Estudo de Casos voltado para o ensino; segundo, o Canadá, juntamente com Estados Unidos, foram o berço dessa metodologia, implantada em suas universidades desde a década de 40 (HERREID, 1994).

Quanto ao uso do método de Estudo de Casos no ensino de Ciências, vimos que tanto o Journal of College Science Teaching, como o Journal Chemical Education, possuem desde 1994 e 1998, respectivamente, uma seção dedicada exclusivamente para a divulgação de trabalhos que relatam estudos voltados para a utilização do método e sua abordagem na sala de aula. 


\section{Análise dos trabalhos encontrados nos periódicos nacionais}

Quantos aos periódicos nacionais, pouquíssimos trabalhos foram encontrados com abordagem do método de estudo de casos, quer voltada para pesquisa, quer para o ensino. Dos 106 trabalhos nacionais encontrados, 59 apresentaram o método de estudo de casos voltados para a pesquisa e apenas 19 trabalhos para o ensino. Vale salientar que a pouca quantidade de trabalhos encontrados que se relacione, de alguma forma, com o método já era um indicativo da baixa presença de trabalhos com abordagem voltada para o tema.

Dos periódicos nacionais, a revista Experiências em Ensino de Ciências foi a que apresentou maior índice de trabalhos que apenas indicam o uso do método, com cerca de 46\% de seus trabalhos, ou seja, sem fazer nenhuma fundamentação metodológica de seu uso, deixando apenas subentendido de que se trata de uma forma de pesquisa. Para quase todos estes periódicos nacionais, com exceção da Química Nova na Escola, os trabalhos que apenas indicavam o uso do método foram ainda maiores do que os que trataram o Estudo de Casos com abordagem para o ensino.

Em relação ao nível de ensino dos trabalhos encontrados, a maioria dos trabalhos apresentaram seus estudos direcionados para a Graduação ou Pós-Graduação, com pouquíssimos trabalhos voltados para o ensino básico. Com relação a essa constatação, Sá e Queiroz (2010) colocam que no ensino de Ciências, o uso de casos é pequeno, no ensino médio, esse número é ainda é menor. Em nossa busca constamos que do total de 147 trabalhos encontrados que abordam o método de estudo de casos como estratégia de ensino, apenas 43 trabalhos, ou seja, 29\%, teve seus estudos voltados para o ensino médio, corroborando mais uma vez com as considerações feitas por Sá e Queiroz (2010).

A seguir realizamos uma Analise Estatística e implicativa (ASI) dos dados, auxiliada pelo software Classification Hiérarchique Implicative et Cohésitive (CHIC), onde foram inseridos todos esses dados.

\section{Análise estatística implicativa dos dados}

\section{Exploração das relações de similaridade}

Para construir a árvore de similaridade, o programa reuniu em uma classe de primeiro nível, primeiramente, as duas variáveis que são mais similares de acordo com o índice de similaridade, depois duas outras variáveis ou uma variável e a classe já formada de acordo com o índice da classe, e depois outras variáveis ou classes de variáveis (COUTURIER, BODIN e GRAS, 2004).

A figura 1 mostra uma árvore hierárquica com os níveis significativos (traços mais grossos e em vermelho), na qual observamos a divisão das 15 variáveis binárias em uma classe que se divide em duas grandes subclasses A e B.

Podemos observar na figura 1, que o aplicativo indica duas grandes classes, que nomeamos de classe A e B. Na classe A, temos as duas variáveis com maior índice de similaridade, são as variáveis estratégia de ensino (V24) e grupo de autores 2 (V28). Estas aparecem no primeiro nível, apresentando maior semelhança que todos os outros pares de variáveis, com um índice de similaridade de 0.99. Essas duas variáveis formam a subclasse que denominamos A2. Observamos ainda que as variáveis: interdisciplinar (V36) que se refere a área de conhecimento e estrangeiro (V9) aparecem no nível 4; apresentando uma 
relação de similaridade de 0.99, formando a subclasse A1. Essa subclasse A1 possui uma relação de similaridade de 0.95 com as variáveis V24 e V28 da subclasse A2. Este resultado vem a confirmar observações indicadas na primeira parte da analise dos dados, uma vez que, para os periódicos estrangeiros (V22) predominam a abordagem do método de estudo de casos voltado para estratégia de ensino cujos autores de referência estão na variável V28 (autores do grupo 2).

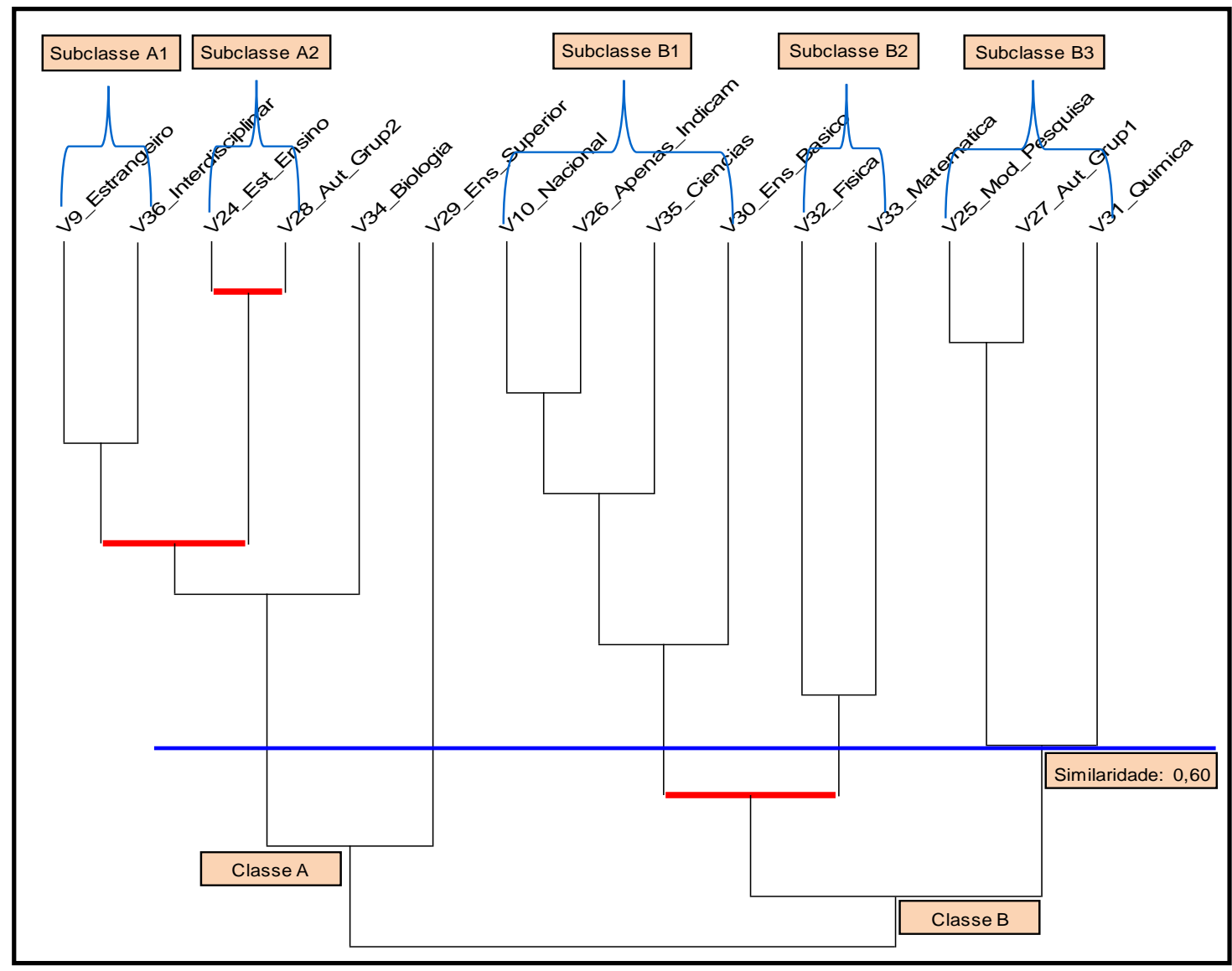

Figura 1. Árvore de similaridade.

A análise das relações de similaridade resultantes da subclasse A demonstra que pelo menos nas amostras dos trabalhos aqui investigados a abordagem do método de estudo de casos voltada para estratégia de ensino apresenta ainda um alto índice de similaridade $(0,89)$ com a área de conhecimento Biologia (V34), sinalizando a existência de uma forte relação de semelhança entre essas variáveis. A árvore também nos mostra uma relação de similaridade desta abordagem com as publicações voltadas para o ensino superior, com um índice de similaridade de 0.54 .

Na classe B, verificamos que as variáveis modalidade de pesquisa (V25) e grupo de autores 1 (V27) estão presentes no segundo nível da árvore, apresentando uma forte relação de semelhança com um índice de similaridade de 0.99. Essas duas variáveis junto com a variável Química (V31), que se refere a área de conhecimento, formam a subclasse B3 com um índice de similaridade de 0.60. Esse baixo índice de similaridade da variável Química com as variáveis (V25) e (V27) está ligado ao fato de não haver muitos trabalhos publicados na área de Química voltados para o tema, de uma forma geral. Ainda analisando a classe B percebemos que no terceiro nível da árvore aparece a variável 'apenas indicam' 
(26) que são os trabalhos que não fundamentaram o método em nenhum dos dois tipos de abordagem, apenas indicaram ter feito uso do mesmo, e a variável 'nacional' (10) que se refere aos periódicos nacionais. De acordo com a árvore de similaridade, essas variáveis apresentam uma forte relação de semelhança entre si com um índice de similaridade de 0.99. Essas duas variáveis juntamente com as variáveis 'Ciências' (V35) e 'ensino básico' (V30) apresentam um índice de similaridade de 0.98 e 0.85 respectivamente, demonstrando existir uma forte relação de semelhança entre elas, formando a subclasse B1.

A árvore nos mostra ainda que as variáveis 'Matemática' (V33) e 'Física' (V32) possuem alguma relação de semelhança entre si com um índice de similaridade de 0.81 formando a subclasse B2. De acordo com nossas pesquisas encontramos pouquíssimos trabalhos relatados na literatura sobre o tema voltado para a área de Matemática e de Física, de acordo com a árvore os poucos trabalhos encontrados nessa área possuem uma relação de similaridade de 0,59 com a subclasse B1.

Ainda de acordo com o gráfico da árvore de similaridade, podemos inferir que a classe B possui algum tipo de relação com um baixíssimo índice de similaridade $\left(8.59742 \mathrm{e}^{-08}\right) \mathrm{com}$ a classe A. Essa relação será mais bem evidenciada pelo gráfico implicativo.

A seguir apresentamos o gráfico implicativo onde é mostrada a organização do conjunto das 36 variáveis estruturado pela relação de implicações com os fechamentos transitivos.

\section{Exploração das relações de implicação}

Para a construção do grafo implicativo (Figura 2) consideramos o valor mínimo de nível de confiança 0,72 que permite apresentar uma estrutura interessante e ainda significativa do ponto de vista estatístico. Lembrando que é possível diminuirmos ainda mais este valor a fim de obtermos mais relações implicativas, porém, quanto menor esse valor, mais fracas se tornam essas relações. Também, do mesmo modo podemos aumentar esse valor para conservarmos as implicações mais fortes.

Após ajustarmos os valores do nível de confiança que ficou em torno de $1-\alpha \geq 0.72$ e $1-\alpha \geq 0.95$ verificamos de acordo com o gráfico, que só 15 variáveis ficaram retidas por ter uma relação de implicação significativa a esses níveis de confiança. Verificamos que dois subconjuntos se destacam nitidamente.

Aos dois subconjuntos que se destacaram após a organização de implicação, chamamos de subconjunto 1 (circulado de vermelho) e subconjunto 2 (circulado de amarelo). Três principais fatores definiram o perfil desses dois grupos, foram eles: a nacionalidade dos periódicos, o nível de ensino e o tipo de abordagem.

No subconjunto 1, aparecem as seis variáveis vinculadas ao perfil que forma o subconjunto 1 que tem por principais fatores que o define as variáveis 'estrangeira', 'estratégia de ensino' e 'ensino superior'. Neste subconjunto, observamos que o aparecimento da variável 'Biologia' (V34) está implicada com um nível de confiança de 0,72 com a variável 'interdisciplinar' (V36). A variável 'interdisciplinar' (V36) encontra-se fortemente implicada $(0,95)$ com a variável 'estrangeiras' (V9) esta, por sua vez, possui uma forte implicação, com um nível de confiança de 0,95, com as variáveis 'estratégia de ensino' (V24) e 'autores do grupo 2' (V27), já estas duas variáveis encontram-se diretamente implicadas com a variável 'ensino superior' (V28) para um nível de confiança de 0,72. 


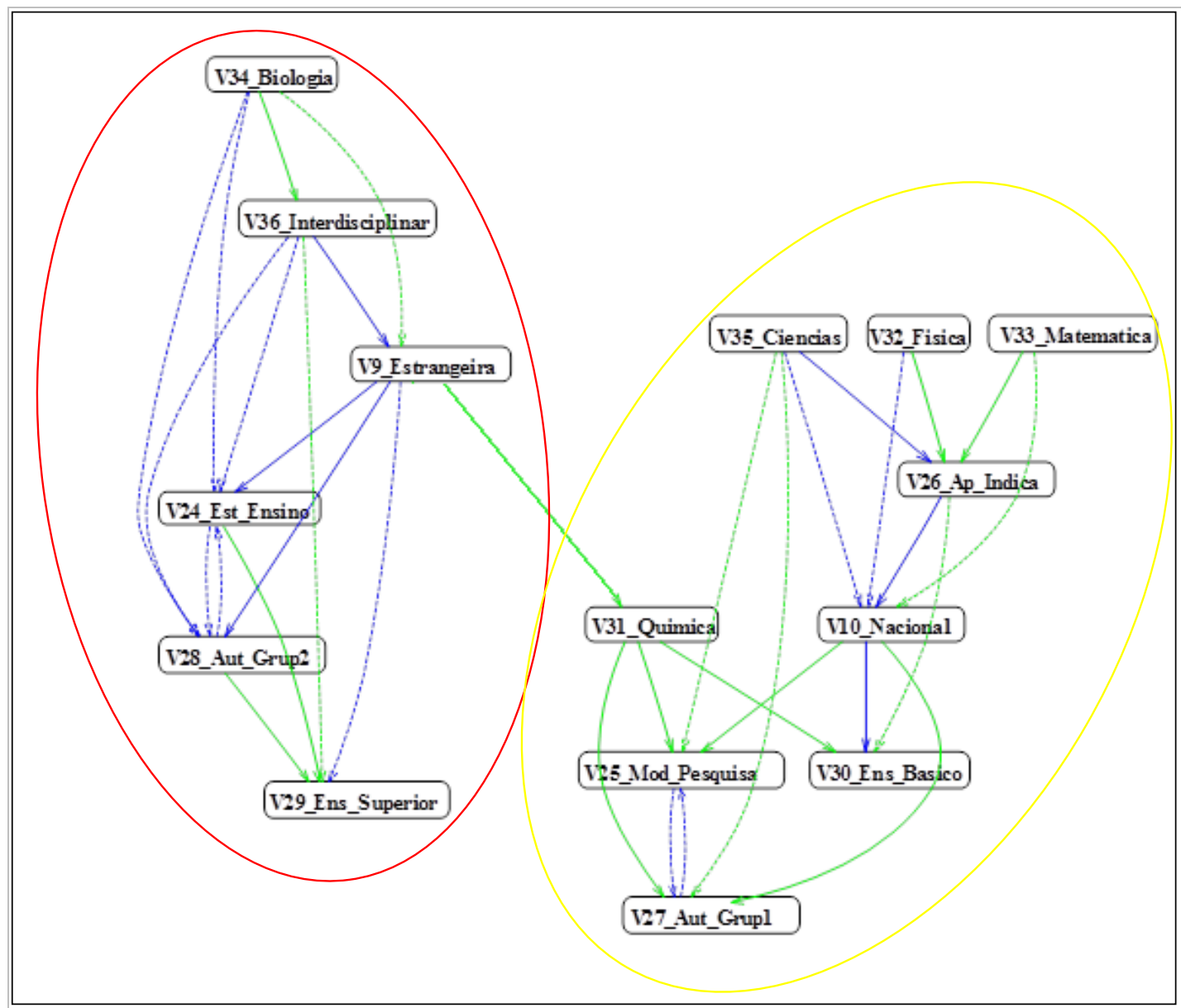

Figura 2. Gráfico implicativo com os fechamentos transitivos.

Estas relações podem evidenciar que as áreas de conhecimento Biologia e interdisciplinar estão mais presentes nos trabalhos que utilizam o método de estudo de casos com uma abordagem voltada a estratégia de ensino, e que este tipo de abordagem do método aparece mais em revistas estrangeiras e no nível superior de ensino. Outra inferência que pode ser feita é que existe uma alta possibilidade dos autores do grupo 2 (Herreid; Conant; Sá e Queiroz; Kortland; Cornely; Cheng; Fortier) estarem citados nos trabalhos das revistas estrangeiras que abordam esse método como estratégia de ensino. Ainda em análise a esse subconjunto, percebe-se que todas as suas relações são transitivamente fechadas e que o mesmo possui uma total ordenação pela relação de implicação.

Em seguida, ao analisarmos o subconjunto 2, aparecem as nove variáveis vinculadas ao perfil que forma esse subconjunto, que tem por principais fatores que o defini as variáveis 'nacional', 'modalidade de pesquisa' e 'ensino básico'. Neste subconjunto observamos que o aparecimento das variáveis 'Ciência' (35), 'Física' (V32) e 'Matemática' (V33) possui uma implicação com um nível de confiança de 0,95, 0,72 e 0,72, respectivamente, com a variável 'apenas indicam' (V26). A variável 'apenas indicam' (V25), por sua vez, encontra-se fortemente implicada $(0,95)$ com a variável 'nacional' (V10). A variável 'Matemática', também aparece implicada com uma relação transitiva a um nível de confiança de 0,78 com a variável 'nacional' (V10). Percebemos ainda que tanto a variável 'nacional' (V10) como a variável 'Química (V31)' possuem uma implicação com um nível de confiança de 0,78 com as variáveis 'modalidade de pesquisa' (V25) e 'autores do grupo 1' (V28), essas duas variáveis 
também encontram-se diretamente implicadas, com um nível de confiança de 0,72, e 0,95 com a variável 'ensino básico' (V29).

Outro fator importante que o gráfico implicativo está nos mostrando é que a variável 'estrangeira' (V9) do subconjunto 1 encontra-se diretamente implicada com a variável 'Química' (V31) do subconjunto 2 com um nível de confiança de 0,72, o que justifica a ocorrência de uma relação de similaridade da classe B com a classe A, mostrada na árvore de similaridade (Figura 1) anteriormente analisada. Em virtude desta constatação podemos inferir que tanto a relação de similaridade entre as classes $A$ e B, como a implicação entre esses dois subgrupos pode estar sendo influenciado pelos periódicos Journal of Chemical Education e Chemistry Education Research Practice, uma vez que são periódicos estrangeiros com publicação exclusiva na área de Química.

A análise dessas relações de implicações presentes no subconjunto 2 pode evidenciar que as áreas de conhecimento Matemática, Física e Ciências estão mais presentes nos trabalhos que apenas indicam do método de Estudo de Caso e também nos trabalhos que utilizam o método com uma abordagem voltada para a modalidade de pesquisa e que estes tipos de abordagem do método aparecem mais em periódicos nacionais e com aplicação no ensino básico. Também podemos inferir a partir dessas relações, que existe uma alta possibilidade dos autores do grupo 1 (Stake; Yin; Bogdan e Biklen; Godoy; Trivinos; Andre e Ludke) estarem citados nos trabalhos dos periódicos nacionais que abordam esse método como modalidade de pesquisa. Uma vez que esses autores compõem o referencial teórico que fundamentam o método com abordagem para a pesquisa científica.

\section{Considerações finais}

Os resultados desta pesquisa demostraram que na área de Educação em Ciências, o método Estudo de Casos tem sido largamente explorado por meio de dois tipos de abordagens: na pesquisa e no ensino.

De acordo com nossa investigação, a literatura estrangeira pesquisada apresentou uma forte tendência de abordagem do método de Estudo de Casos como estratégia de ensino, em contra partida, a literatura brasileira, além de ter mostrado um baixo índice de publicações relacionadas com o tema, apresentou na maioria de seus trabalhos uma forte tendência de abordagem do método de Estudo de Casos como modalidade de pesquisa.

No contexto da educação em Química, os resultados apontaram uma fraca tendência de abordagem do método de Estudo de Casos como estratégia de ensino, tanto na literatura estrangeira, como na literatura brasileira. O Chemistry Education Research Practice, que também é exclusivo da área de Química, apresentou uma maior tendência de abordagem do método voltada para a pesquisa, mostrando a possibilidade de na literatura estrangeira haver certa incipiência de abordagem do método voltada para o ensino, na área da Química. Corroborando com esse resultado, o Journal College Science Teaching, que é um periódico que abrange todas as áreas ligadas ao ensino das Ciências e que apresentou 99\% de seus trabalhos com o método voltado para o ensino, menos de $9 \%$ destinou-se a área de Química.

De acordo com a literatura brasileira, a introdução do Estudo de Casos com abordagem para o ensino na Educação em Ciências, em geral, ainda tem sido um tanto lenta. 
Quanto ao nível de ensino constatamos que, tanto na literatura estrangeira como na brasileira, o uso do Estudo de Casos tem ganhado maiores proporções na graduação. Sua utilização, por outro lado, tem se mostrado ainda um tanto tímida na educação básica.

A realização da análise estatística nos possibilitou observar importantes relações hierárquicas implicativas nas formas de utilização do método do Estudo de Casos nos trabalhos investigados, onde podemos identificar de forma mais consistente não só as áreas de conhecimento que mais estão presentes nos trabalhos que utilizam o Estudo de Casos com abordagem voltada, tanto para o ensino, como para a pesquisa, como também, os níveis de ensino em que essas abordagens têm sido mais empregadas. Também nos permitiu conhecer as contribuições de cada periódico para os resultados apontados nesta investigação.

Finalmente, os resultados desta pesquisa contribuem para um maior enriquecimento da literatura que estuda a utilização do método de Estudo de Casos como estratégia pedagógica na área da Educação em Ciências. No ensino de Química o estudo realizado sugere a necessidade de implementação de práticas pedagógicas que utilizem a metodologia de Estudo de Casos em diferentes níveis de ensino.

\section{Referências}

ALMOULOUD, S. A.; GRAS, R.; RÉGNIER, J.-C. A.S.I. - Análise estatística implicativa: mais uma vez, o que é? Educ. Matem. Pesq., v.16, n.3, p.623-1087, 2014.

ANDRÉ, M.E.D.A. Estudo de Caso: Seu Potencial na Educação. Cadernos de Pesquisa, n.49, p.51-54, 1984.

ANDRÉ, M. E. D. A. (1998). Etnografia da prática escolar. $2^{a}$ ed. São Paulo: Papirus Editora, 1998.

BARBOSA, F. T.; AIRES, J. A. Visões sobre natureza da ciência em artigos publicados em periódicos nacionais da área de ensino de ciências: um olhar para a educação em química.

Amazônia - Revista de Educação em Ciências e Matemática, v.14, n. 30, p. 77-104, Jan-Jul, 2018.

BOAVENTURA, E. M. Metodologia da Pesquisa: Monografia, dissertação e tese. São Paulo: Atlas, 2014.

BOCCHI, S.C.M.; PESSUTO, J.; DELL'AQUA, M.C.Q. Modelo operacional do estudo de caso como estratégia de ensino na disciplina de enfermagem médico-cirúrgica: avaliação dos alunos. Rev.latino-am.enfermagem, v. 4, n. 3, p. 99-116, 1996.

CARNIELLO, M. F.; ZULIETTI, L. F. Métodos Pedagógicos em Cursos de Comunicação Social: Aplicação e Formação de Banco de Casos. UNOPAR Cient., Ciênc. Human. Educ., v. 16, n.4, p. 278-289, 2015.

CLEMENTE-JÚNIOR, S. dos S. Estudo de Caso x Casos para Estudo: Esclarecimentos a cerca de suas características e utilização. In.: Anais do VII Seminário de pesquisa em turismo do Mercosul. Universidade de Caxias do Sul. Caxias do Sul - Rio Grande do Sul, 2012.

CONANT, J. B. Dois modos de pensar. Tradução de Anísio Teixeira. São Paulo: Companhia Editora Nacional: Editora Universidade de São Paulo, 1968. 
COUTURIER R, BODIN A, GRAS R. A classificação hierárquica implicativa e coesiva. Manual Curso CHIC versão 2.3; 2004. Disponível:

http://math.unipa.it/ grim/asi/asi 03 gras bodin cout.pdf. Acessado em setembro de 2015.

ELLET, W. Manual de estudo de caso: como ler, discutir e escrever casos de forma persuasiva. Tradução: André de Godoy Vieira. Porto Alegre: Bookman, p. 270, 2008.

GRAS R, ALMOULOUD SA. A implicação estatística usada como ferramenta em um exemplo de análise de dados multidimensionais. In: I Colóquio o Método Estatístico Implicativo Utilizado em Estudos Qualitativos de Regras de Associação Contribuição à Pesquisa em Educação. São Paulo, 2003. Disponivel em:

http://math.unipa.it/ grim/asi/asi_03_saddo_gras.pdf.

HERREID, C. F. Using Case Studies to Teach Science. Actionbioscience. 2005.

http://www.actionbioscience.org/education/herreid.html.

HERREID, C. F. Case studies in science - a novel method of science education. Journal of College Science Teaching, v. 23, n. 4, p. 221-229, 1994.

HERREID, C.F. What is a case? Journal of College Science Teaching, 27, 2, 92-94, 1997.

IKEDA, A. A.; VELUDO-DE-OLIVEIRA, T. M.; CAMPOMAR, M. C. A tipologia do método do caso em administração: usos e aplicações. O\&S, v. 12, n. 34, 2005.

JONASSEM D. H. Contributing Editor, Typology of case based Learning: The Content, Form and Function of case - Education Technology. July/ Aug., 2006.

KORTLAND, K. An STS case study about students' decision making on the waste issue.

Science Education, v. 80, n. 6, p. 673-689, 1996.

LERMAN, I. C. Classification et Analyse Ordinale des Données, Dunod, 1980.

REIS, E. M.; LINHARES, M. P. Integrando o espaço virtual de aprendizagem "Eva" à formação de professores: estudo de caso sobre o currículo de Física no ensino médio. ENSAIO Pesquisa em Educação em Ciências, v. 10, n.2, p. 1-22, 2008.

MARTINS, G. A. Estudo de caso: uma reflexão sobre a aplicabilidade em Pesquisas no Brasil. RCO - Revista de Contabilidade e Organizações - FEARP/USP, v. 2, n. 2, p. 8 - 18 jan./abr. 2008 .

MENEZES, M. A. A. Do método do caso ao case: a trajetória de uma ferramenta pedagógica. Educação e Pesquisa, v.35, n.1, p. 129-143, 2009.

MOREIRA, M. M. (2011). Metodologias de pesquisa em ensino. $1^{a}$ edição, São Paulo: Editora Livraria da Física, 2011.

PINHEIRO, A.N.; MEDEIROS, E.L. e OLIVEIRA, A.C. Estudo de casos na formação de professores. Química Nova, v. 33, n. 9, 1996-2002, 2010.

REIS, P. O ensino de ética nas aulas de ciências através do estudo de casos. Interacções, n. 5, p. 36-45, 2007.

SÁ, L. P. A argumentação no ensino superior de química: investigando uma atividade fundamentada em estudos de casos. 2006. 153 p. Dissertação de Mestrado, Universidade de São Paulo, Brasil. 
SÁ, L. P.; QUEIROZ, S. L. Estudo de casos no Ensino de Química. Campinas: Editora Átomo, 2010.

SILVA, R. R.; BENEGAS, A. A. O uso do estudo do caso como método de ensino na graduação. Economia \& Pesquisa, v. 12, n.12, p. 9 - 31, 2010.

VASCONCELOS, Y. L; YOSHITAKE, M; LINS, D. A. C; MELO, A. P. S. de; FRANÇA, S. M. de. Método de caso e estudo de caso: usos no exercício da docência em contabilidade de custos. Custos e @gronegócio on line,v. 9, n. 4, 2013. Disponível em:

http://www.custoseagronegocioonline.com.br/numero4v9/Metodo.pdf. Acessado em: abril de 2017.

YIN, R. K. Estudo de Caso, planejamento e métodos. 2.ed. São Paulo: Bookman, 2001.

YIN, Robert K. Case study research: design and methods. Newbury Park, CA: Sage

Publications, 1989. 